\title{
An analysis of the optical spectra of the post-asymptotic giant branch stars LSIV -12111 and HD 341617
}

\author{
R. S. I. Ryans ${ }^{1}$, P. L. Dufton ${ }^{1}$, C. J. Mooney ${ }^{1}$, W. R. J. Rolleston ${ }^{1}$, F. P Keenan ${ }^{1}$, I. Hubeny ${ }^{2,3}$, and T. Lanz ${ }^{2,4}$ \\ 1 The Department of Pure and Applied Physics, The Queen's University of Belfast, Belfast BT7 1NN, Northern Ireland \\ 2 Laboratory for Astronomy and Solar Physics, Code 681, Goddard Space Flight Centre, Greenbelt, MD 20771, USA \\ 3 National Optical Astronomy Observatories, Tucson, AZ 85726, USA \\ ${ }^{4}$ Department of Astronomy, University of Maryland, College Park, MD 20742, USA
}

Received 23 August 2002 / Accepted 4 February 2003

\begin{abstract}
High spectral resolution and signal-to-noise observations of the absorption and emission line spectra in two postasymptotic-giant-branch (PAGB) stellar candidates, LSIV -12 111 and HD 314617 are discussed. The absorption line spectra have been analysed using non-LTE model atmosphere techniques to determine stellar atmospheric parameters and chemical compositions, both in absolute terms and relative to a standard star, HD 13841. The atmospheric parameters differ from previous estimates based on LTE model atmospheres, probably due to non-LTE effects. In turn these imply stellar masses that are generally larger than have been previously estimated. Both PAGB candidates have relative uniform underabundances of metals with mean values of -0.35 dex for LSIV -12111 and -0.50 dex for HD 314617.

Furthermore, their abundance patterns are remarkably similar to that observed for optically bright, F-type PAGBs. From the emission spectra, the plasma parameters and angular size of the circumstellar material are constrained, and these are consistent with previous estimates and with a PAGB evolutionary stage.
\end{abstract}

Key words. stars: abundances - stars: supergiants - stars: early-type - stars: fundamental parameters

\section{Introduction}

Stars evolving from the asymptotic giant branch to become planetary nebulae pass through a short lived but important evolutionary stage that is designated as post-asymptotic giant branch (PAGB). The properties of PAGB stars have been reviewed, for example, by Kwok (1993) and by Hrivnak (1997). This evolutionary stage is important both in its own right, for the insight it provides on nucleosynthetic and mixing processes within AGB stars, and because such stars form an important component of globular clusters. For example, PAGB candidates can exhibit a wide variety of morphologies ranging from heavily obscured objects (such as Roberts 22 - Sahai et al. 1999; Egg Nebula - Sahai et al. 1998; IRAS 17150-3224 - Kwok et al. 1998; IRAS 17441-2411 - Su et al. 1998), to relatively unobscured late-type (see, for example, Reddy et al. 2002; Luck \& Bond 1991) and early-type (Rauch et al. 2002; Moehler \& Heber 1998) stars. Most PAGB candidates have been identified from their infra red colours and magnitudes (see, for example, Oudmaijer et al. 1992, 1995; Oudmajier 1996) and have also been observed in the $\mathrm{mm}$ and radio spectral regions (Dyer et al. 2001; Gledhill et al. 2002; Evans et al. 2002). The stellar spectra, when they can be directly observed, show a wide variety of chemical compositions with, for example, both

Send offprint requests to: R. Ryans,

e-mail: R.Ryans@qub.ac.uk carbon rich (Reddy et al. 2002; Van Winckel \& Reyniers 2000) and apparantly carbon deficient (Conlon et al. 1993a; Moehler \& Heber 1998) objects having been identified. Probably their most interesting characteristic is that their very short evolutionary timescales allow evolutionary changes to be observed directly (Parthasarthy et al. 2000), while related objects such as V4334 Sgr (the "Sakurai" object) appear to be "born again" stars that have rapidly evolved following a helium flash from a white dwarf to a PAGB regime (Asplund et al. 1999; Evans et al. 2002). PAGB stars have been unambiguously identified in globular clusters (Landsmann et al. 1998), where they have the potential to act as standard candles (Alves et al. 2001). Their surface chemical composition often differs significantly from that of the underlying cluster (Mooney et al. 2001; Moehler et al. 1998), with dust gas separation likely to be an important mechanism.

During a project to identify and characterize normal B-type stars in the Galactic Halo (see, for example, Magee et al. 2001), a significant number of subluminous evolved stars have been identified. Most of these targets have been classified as posthorizontal branch (PHB) but a significant number appear to be PAGB (see, for example, Hambly et al. 1997). One particularly interesting object is LSIV - 12111 , which was classified from low dispersion spectroscopy by Kilkenny \& Pauls (1990) as having a spectral type around B0. From model atmosphere techniques, McCausland et al. (1992, hereafter MCDK92) 
concluded that the chemical composition and atmospheric parameters of LSIV -12111 were consistent with a PAGB evolutionary state. This was supported by Conlon et al. (1993a, hereafter CDMK93, 1993b) who examined its observed nebular emission and infrared excess. The spectroscopic data available for these studies were not of sufficient quality to reliably identify and measure the weak absorption and emission lines in LSIV - 12 111. We have therefore obtained optical spectra of LSIV -12 111 at a high spectral resolution and with a better signal-to-noise ratio with the primary objective of better constraining its evolutionary status.

Recently, while undertaking spectroscopic observations at the Keck telescopes, another PAGB stellar candidate, HD 341617, was observed. Moderate spectral resolution observations of this star have been previously discussed by Parthasarthy et al. (2000), who identified it as being at a PAGB evolutionary stage. Indeed, as discussed by Parthasarthy et al. and confirmed by the current higher resolution spectroscopy, the absorption spectra of the two stars (and in particular the silicon and the Balmer lines) implied that they have similar atmospheric parameters, whilst their emission spectra implied that the circumstellar environments are similar (see Fig. 1).

Here we analyse the absorption line spectra of both stars using non-LTE model atmosphere techniques. Additionally we use the emission line spectra to constrain the properties of their circumstellar environments.

\section{Observations and data reduction}

\section{1. $L S I V-12111$}

Spectra of LSIV -12 111 were obtained using the CASPEC spectrograph on the ESO 3.6m in August 1994. A $512 \times 512$ pixel Tektronix CCD was used with a slit width of 1.0 arcsec, and a total of nine $20 \mathrm{~min}$ exposures were made over two nights. Complete wavelength coverage was obtained from 3700-5100 $\AA$ with the mean continuum signal-to-noise ratios (SNRs) for the resultant summed spectra typically in excess of 200.

Data reduction for the LSIV -12 111 observations was similar to that described in Lennon et al. (1996). Briefly, the data were reduced to a one dimensional format using standard procedures to flat field and de-bias the images within IRAF (Massey 1989; Shames \& Tody 1986). Cosmic ray events were removed using a combination of automated routines and manual inspection, while wavelength calibration were performed against interleaved exposures of a Th-Ar lamp.

\section{2. $H D 341617$}

Spectroscopic data for HD 341617 were obtained with the $10-\mathrm{m}$ Keck I telescope on 4 July 2000. The HIRES echelle spectrometer (HIRESb) was employed with the 53 grooves $\mathrm{mm}^{-1}$ grating and a slit width of 1.0 arcsec. This gave complete wavelength coverage from approximately 3650 to $5170 \AA$ at a resolution $(F W H M)$ of $R \approx 40000$. Typical continuum counts of 30000 were achieved resulting in a signalto-noise ratio of approximately 170 . Data reduction again

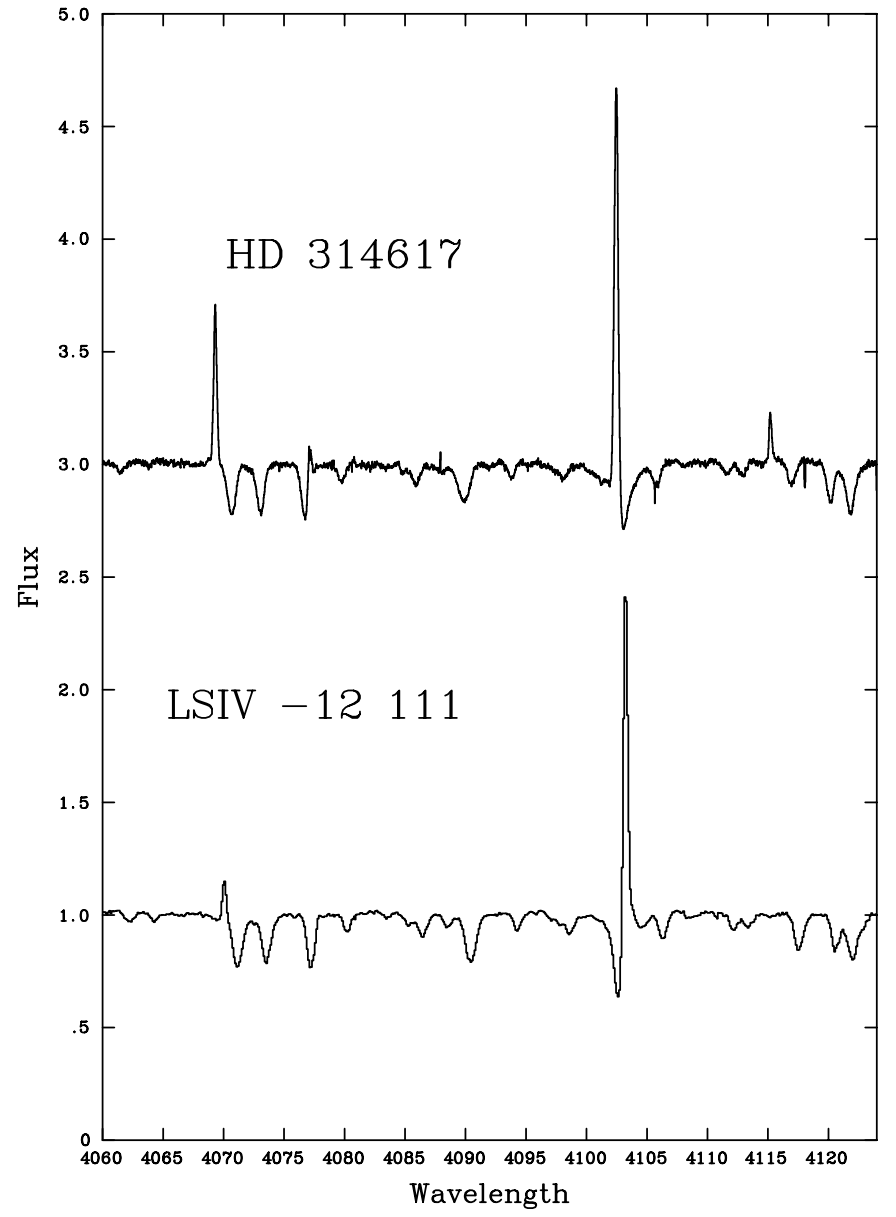

Fig. 1. Spectra of the two PAGB candidates, LSIV -12111 and HD 341617, showing the similarity of their absorption and emission line spectra.

Table 1. Heliocentric radial velocity estimates for the programme stars.

\begin{tabular}{lllll}
\hline \hline Star & Spectrum & Source & $\begin{array}{l}\text { Observation } \\
\text { date }\end{array}$ & $\begin{array}{c}v_{\mathrm{r}} \\
\mathrm{km} \mathrm{s}^{-1}\end{array}$ \\
\hline LSIV-12111 & Absorption & ESO & 26 Aug. 94 & $83 \pm 6$ \\
& & ESO & 27 Aug. 94 & $97 \pm 6$ \\
& & AAT & 13 Aug. 89 & $97 \pm 5$ \\
LSIV-12111 & Emission & ESO & 26 Aug. 94 & $93 \pm 2$ \\
& & ESO & 27 Aug. 94 & $93 \pm 2$ \\
& & AAT & 13 Aug. 89 & $92 \pm 1$ \\
HD 341617 & Absorption & Keck & 4 Jul. 00 & $64 \pm 2$ \\
HD 341617 & Emission & Keck & 4 Jul. 00 & $51 \pm 2$ \\
\hline
\end{tabular}

followed standard procedures and further details can be found in Mooney et al. (2002).

\section{3. $H D 13841$}

Spectra of a standard star HD 13841 were obtained in July 2000, on the $4 \mathrm{~m}$ William Herschel Telescope using the UES and a slit width of 0.5 arcsec. Two $2048 \times 4096$ pixel EEV CCDs were used as the detectors and wavelength coverage from 3700 to $6400 \AA$ was obtained, with a mean continuum 
$S N R \approx 200$ in the regions of interest. Data reduction procedures were analogous to those used for LSIV -12111 .

\subsection{Measurement of radial velocities and equivalent widths - absorption lines}

Metal and non-diffuse helium lines in both stars were examined using the STARLINK package DIPSo (Howarth et al. 1994). The spectra were normalized by fitting low order polynomials to the continuum regions, and Gaussian profiles were then fitted to the lines using non-linear least squares fitting routines, providing measurements of line centroids and equivalent widths. The former were then used to deduce heliocentric radial velocities $\left(v_{\mathrm{r}}\right)$ which are summarized in Table 1.

For LSIV - 12111 , these implied a change in radial velocity between the observations obtained on successive nights in the absorption lines only, and this was confirmed by a cross correlation technique to be $\Delta v_{\mathrm{r}}=14 \pm 3 \mathrm{~km} \mathrm{~s}^{-1}$. The emission line spectrum shows no evidence of such time-dependent behavior (see Sect. 2.5), confirming that the shift seen in the absorption centroids is not an artifact of the reduction process, but a real physical effect. Also listed in Table 1 is the heliocentric velocity for LSIV -12 111 obtained by CDMK93 from the absorption lines.

As discussed in Sect. 2.5, the emission line spectrum is similar to that found in low ionization planetary nebula. Additionally the relative strengths of these lines can be used to estimate the plasma parameters (see Sect. 3.2). The estimated electron density, $N_{\mathrm{e}} \simeq 4 \times 10^{3} \mathrm{~cm}^{-1}$, is again compatible with that found in planetary nebulae as is the electron temperature estimate of $7800 \mathrm{~K}$; for example, Hyung et al. (2000) estimated $N_{\mathrm{e}} \simeq 5 \times 10^{3} \mathrm{~cm}^{-1}$ and $T_{\mathrm{e}}=8000-8300 \mathrm{~K}$ for the planetary nebula, NGC 6543 . Hence we believe that the emission line spectrum is due the presence of circumstellar material, which is starting to ionize as LSIV -12 111 evolves to higher temperatures and becomes a planetary nebula. In particular, the species observed (including many forbidden transitions), the plasma parameters, and the intrinsic narrowness of the lines all seem incompatible with the emission lines being formed in a stellar wind. The variation in radial velocity deduced from the absorption lines would then seem to imply that LSIV -12 111 is a single lined spectroscopic binary (see Sect. 4.3).

The spectroscopic data for HD 341617 were all obtained on the same night and cross correlation techniques showed no significant shifts in the absorption lines between these exposures, with the heliocentric radial velocities again being summarized in Table 1. Additionally, Parthasarathy et al. (2000) deduced an absorption line radial velocity of $46 \pm 5 \mathrm{~km} \mathrm{~s}^{-1}$ from their INT observations obtained in July/August 1993 and state that they "do not show any signature for binarity and symbiotic type". It would appear that the radial velocity of Parthasarathy et al. (2000) is geocentric and transforming to a heliocentric frame (to be consistent with Table 1) would imply a value of $35 \pm 5 \mathrm{~km} \mathrm{~s}^{-1}$. Hence there may be evidence for a radial velocity change between the two sets of observations.

In order to obtain the highest quality absorption spectra possible for LSIV -12111 , the observations were arbitrarily moved to a common rest frame by first applying a velocity shift of $14 \mathrm{~km} \mathrm{~s}^{-1}$ to the first night's data, and then coadding the resulting spectra. This procedure does, of course, "smear out" the time-independent emission lines in the coadded spectra, but those were not of interest at this point in the analysis.

Equivalent widths for the metal and non-diffuse helium absorption lines in the two programme stars were estimated using the fitting procedures discussed above. For sets of unresolved (or partially resolved) lines, the fitting procedure explicitly included all the components but only a total equivalent width was estimated. These equivalent widths are too extensive to reproduce here but are available from the CDS website.

For the diffuse helium lines and the hydrogen lines $\mathrm{H}_{\gamma}, \mathrm{H}_{\delta}$, and $\mathrm{H}_{\epsilon}$, the spectra were normalized and profiles measured, with the continuum levels being defined at $\pm 16 \AA$ from the line centre for both observational data and the subsequent theoretical analyses.

\subsection{Measurement of radial velocities and equivalent widths - emission lines}

As discussed in CDMK93, LSIV - 12111 displays strong emission lines in the Balmer series and also forbidden lines of [S II] and [N II]. The Keck spectra for HD 341617 also showed an extensive emission line spectrum again with strong emission in the Balmer series, in agreement with the observations discussed by Parthasarathy et al. (2000).

A cross correlation of the emission line spectra from subsequent nights of ESO observations for LSIV - 12111 showed no evidence of a velocity shift, a finding confirmed by fitting individual emission profiles with a Gaussian profile and comparing the measured centroids. The radial velocity estimates obtained using these methods, listed in Table 1, are in good agreement with those of MCDK92 for LSIV - 12 111, indicating the absence of long term radial velocity variation in the emission spectra. It was therefore reasonable to directly co-add the two nights of ESO data, and then using the emission line velocity in Table 1 to bring the spectrum to a laboratory rest frame.

For HD 341617, there was again no evidence for any shift in the emission line spectrum for the short time cadence (approximately 3 hours) of the observations. Additionally, we found no evidence for the "large scatter" and "weak correlation between optical depth and radial velocity" suggested by Parthasarthy et al. (2000). Indeed the standard deviation of $\pm 2 \mathrm{~km} \mathrm{~s}^{-1}$ found in our measurements implies that any effects would appear to be small. The spectra were therefore simply coadded and individual emission lines fitted to obtain the heliocentric velocity listed in Table 1, which in turn was used to shift the spectrum to the rest frame.

The coadded emission spectra were then searched for weaker emission lines, and approximately 60 were identified. Again, a non-linear least squares fitting routine was employed to estimate the centroids of the feature and the emission line equivalent width relative to the normalized stellar continuum $\left(W_{\mathrm{e}}\right)$. These are summarized in Table 2 . Note that although the Balmer series was observed in emission up to 
approximately $\mathrm{H}_{13}$, results are only presented up to $\mathrm{H}_{8}$. This reflects the difficulty in disentangling the emission from the underlying stellar absorption. A simple approach was adopted for the hydrogen lines - the stellar absorption was assumed to be symmetric, and since the emission lies in the wings of the absorption line, the stellar profile was taken from the other wing and mirrored onto the wavelength region containing emission. Numerical experiments suggest that the uncertainties due to this process (i.e. blending in the other wing and emission spreading into the line centre and other wing) were of the order of at least $10 \%$, and increased along the series, leading us to limit our measurements to the lower series members.

Approximately eighty percent of all of the features could be identified using line lists for planetary nebulae and $\mathrm{H}$ II regions (Aller \& Hyung 1995; Hyung et al. 1994; Hyung et al. 1995; Osterbrock et al. 1992) and for the symbiotic star RR Tel (McKenna et al. 1997). Identifications for the remaining features utilised the web based line lists of van Hoof (at http://www.pa.uky.edu/ peter/atomic/). In most cases the identification appeared clear cut, but in four cases (marked with :) they were less secure. As may be seen from Table 2, the spectra are dominated by forbidden lines due to $[\mathrm{Fe}$ II $]$ and [ $\mathrm{Fe}$ III] together with allowed transitions in $\mathrm{H}_{\text {I }}, \mathrm{C}_{\text {II }}$ and Si II.

\section{Analysis}

\subsection{Model atmosphere analysis of absorption line spectra}

In contrast to our previous work on B-type supergiant stars (see, for example, McErlean et al. 1999), which used the DETAIL and SURFACE (Butler 1984; Giddings 1981) model atmosphere codes, we here adopt the TLUSTY and SYNSPEC packages (Hubeny 1988; Hubeny \& Lanz 1995; Hubeny et al. 1998). TLUSTY calculates plane-parallel, horizontally homogeneous model stellar atmospheres in radiative and hydrostatic equilibrium, and provides for fully consistent, non-LTE metal line blanketing. The companion program SYNSPEC then calculates the emergent spectrum for a given model atmosphere.

The metal line blanketing features of TLUSTY operate by constructing a model including the key chemical species that determine the atmospheric structure; typically $\mathrm{H}, \mathrm{He}, \mathrm{C}, \mathrm{N}, \mathrm{O}$, $\mathrm{Mg}, \mathrm{Si}, \mathrm{Fe}$, and $\mathrm{Ni}$, with the latter two elements being the dominant opacity source in most cases. Due to limits on the available computational power our models did not include $\mathrm{Ni}$, but experience has shown that the bulk of the opacity in the regime of interest is due to Fe (Hubeny et al. 1998) so this simplification is unlikely to be a source of significant error. In addition, our technique of differential analysis should ensure that any systematic errors in our approach should largely cancel out. We believe the adoption of TLUSTY represents a significant advance in the sophistication of our models, with the ability to incorporate consistent line blanketing, and easily run multi-element models. For the analysis discussed here we used TLUSTY version 198D. The default configuration was mostly adopted, apart from in the case of microturbulence. By default TLUSTY includes the effects of microturbulence in both the variation of line profiles and also via a turbulent pressure term. We opted to disable
Table 2. Emission line equivalent widths in LSIV -12111 and HD 341617.

\begin{tabular}{|c|c|c|c|c|c|}
\hline \multicolumn{2}{|c|}{ Identification } & \multicolumn{2}{|c|}{$\overline{\mathrm{LSSIV}-12111}$} & \multicolumn{2}{|c|}{ HD 341617} \\
\hline Species & $\lambda(\AA)$ & $\lambda(\AA)$ & $W_{\mathrm{e}}(\mathrm{m} \AA)$ & $\lambda(\AA)$ & $W_{\mathrm{e}}(\mathrm{m} \AA)$ \\
\hline$\overline{\mathrm{Si}}$ II & 3856.02 & 3856.00 & 39 & 3856.01 & 117 \\
\hline Si II & 3862.59 & 3862.61 & 31 & 3862.57 & 64 \\
\hline$\left[\mathrm{O}_{\text {II }}\right]$ & 3726.03 & 3726.03 & 790 & 3726.03 & 83 \\
\hline [O II $]$ & 3728.82 & 3728.79 & 392 & 3728.80 & 28 \\
\hline $\mathrm{He}_{\mathrm{I}}$ & 3888.65 & & & 3888.69 & 144 \\
\hline $\mathrm{H}_{\mathrm{I}}$ & 3889.05 & 3888.97 & 520 & 3889.04 & 280 \\
\hline $\mathrm{H}_{\mathrm{I}}$ & 3970.07 & 3970.07 & 600 & 3970.07 & 406 \\
\hline [Ni II] & 3993.15 & & & 3993.07 & 74 \\
\hline$\left[\mathrm{S}_{\mathrm{II}}\right]$ & 4068.60 & 4068.57 & 57 & 4068.58 & 200 \\
\hline $\mathrm{H}_{\mathrm{I}}$ & 4101.76 & 4101.74 & 1040 & 4101.72 & 982 \\
\hline$[\mathrm{Fe}$ II $]$ & 4114.48 & & & 4114.46 & 54 \\
\hline$[\mathrm{Cr} \mathrm{II}]$ & 4164.63: & 4164.67 & 60 & 4164.56 & 17 \\
\hline$\left[\mathrm{Fe}_{\mathrm{II}}\right]$ & 4177.21 & & & 4177.18 & 73 \\
\hline$[\mathrm{Fe}$ II $]$ & 4178.95 & & & 4178.93 & 27 \\
\hline [Ni II] & 4201.74 & & & 4201.83 & 36 \\
\hline$[\mathrm{Fe}$ II $]$ & 4211.10 & & & 4211.10 & 29 \\
\hline$\left[\mathrm{Fe}_{\mathrm{II}}\right]$ & 4243.98 & 4243.89 & 25 & 4243.95 & 284 \\
\hline$[\mathrm{Fe} \mathrm{II}]$ & 4244.81 & & & 4244.79 & 66 \\
\hline$\left[\mathrm{V}_{\text {III }}\right.$ & 4248.81: & & & 4248.81 & 36 \\
\hline$\left[\mathrm{Fe}_{\mathrm{II}}\right]$ & 4251.49 & & & 4251.41 & 16 \\
\hline $\mathrm{C}_{\text {II }}$ & 4267.18 & 4267.18 & 31 & Blended & \\
\hline$[\mathrm{Fe} \mathrm{II}]$ & 4276.83 & & & 4276.81 & 258 \\
\hline$\left[\mathrm{Fe}_{\mathrm{II}}\right]$ & 4287.40 & 4287.36 & 44 & 4287.37 & 701 \\
\hline$\left[\mathrm{Fe}_{\mathrm{II}}\right]$ & 4305.90 & & & 4305.87 & 68 \\
\hline$[\mathrm{Fe}$ II $]$ & 4319.53 & & & 4319.59 & 132 \\
\hline [Ni II] & 4326.28 & & & 4326.25 & 83 \\
\hline $\mathrm{H}_{\mathrm{I}}$ & 4340.46 & 4340.46 & 1980 & 4240.47 & 2260 \\
\hline$[\mathrm{Fe} \mathrm{II}]$ & 4352.78 & & & 4352.75 & 145 \\
\hline$[\mathrm{Fe} \mathrm{II}]$ & 4358.37 & & & 4358.33 & 123 \\
\hline$[\mathrm{Fe}$ II $]$ & 4359.34 & 4359.31 & 32 & 4359.30 & 519 \\
\hline $\mathrm{C}_{\text {II }}$ & 4368.14 & 4368.23 & 24 & 4368.21 & 148 \\
\hline$[\mathrm{Fe}$ II $]$ & 4372.43 & & & 4372.41 & 78 \\
\hline$\left[\mathrm{Fe}_{\mathrm{II}}\right]$ & 4382.75 & & & 4382.72 & 47 \\
\hline$[\mathrm{Fe}$ II $]$ & 4413.78 & 4413.72 & 20 & 4413.75 & 377 \\
\hline$[\mathrm{Fe} \mathrm{II}]$ & 4416.27 & & & 4416.23 & 374 \\
\hline$[\mathrm{Fe}$ II $]$ & 4419.59 & 4419.59 & 26 & 4419.56 & 20 \\
\hline$[\mathrm{Fe} \mathrm{II}]$ & 4457.95 & 4457.94 & 4 & 4457.92 & 246 \\
\hline [Fe III] & 4474.91 & 4474.91 & 9 & 4474.87 & 126 \\
\hline$[\mathrm{Fe} \mathrm{II}]$ & 4488.75 & & & 4488.72 & 100 \\
\hline$[\mathrm{Fe} \mathrm{II}]$ & 4492.61 & & & 4492.60 & 59 \\
\hline$[\mathrm{Fe}$ II $]$ & 4509.61 & & & 4509.58 & 24 \\
\hline$[\mathrm{Fe} \mathrm{II}]$ & 4514.90 & & & 4514.87 & 56 \\
\hline$\left[\mathrm{Fe}_{\mathrm{II}}\right]$ & 4528.38 & & & 4528.35 & 28 \\
\hline [Ni II] & 4628.04 & & & 4628.04 & 31 \\
\hline [Fe III] & 4658.10 & 4658.10 & 10 & 4658.13 & 117 \\
\hline [Fe III] & 4701.62 & & & 4701.56 & 43 \\
\hline$\left[\mathrm{Fe}_{\mathrm{II}}\right]$ & 4728.07 & & & 4728.05 & 184 \\
\hline [Fe III] & 4733.93 & & & 4733.90 & 24 \\
\hline$[\mathrm{Fe} \mathrm{III}]$ & 4754.70 & & & 4754.73 & 20 \\
\hline [Fe III] & 4769.60 & & & 4769.55 & 18 \\
\hline$\left[\mathrm{Fe}_{\mathrm{II}}\right]$ & 4774.74 & & & 4774.69 & 131 \\
\hline [Fe III] & 4777.88 & & & 4777.71 & 8 \\
\hline$[\mathrm{Fe}$ II $]$ & 4814.55 & 4814.54 & 21 & 4814.51 & 414 \\
\hline $\mathrm{HI}_{\mathrm{I}}$ & 4861.33 & 4861.32 & 5840 & 4861.36 & 6290 \\
\hline$[\mathrm{Fe} \mathrm{II}]$ & 4874.49 & & & 4874.47 & 141 \\
\hline [Fe III] & 4881.00 & & & 4881.02 & 28 \\
\hline$\left[\mathrm{Fe}_{\mathrm{II}}\right]$ & 4889.63 & 4889.56 & 17 & 4889.58 & 335 \\
\hline
\end{tabular}


Table 2. continued.

\begin{tabular}{|c|c|c|c|c|c|}
\hline \multicolumn{2}{|c|}{ Identification } & \multicolumn{2}{|c|}{ 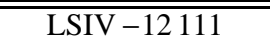 } & \multicolumn{2}{|c|}{ HD 341617} \\
\hline Species & $\lambda(\AA)$ & $\lambda(\AA)$ & $W_{\mathrm{e}}(\mathrm{m} \AA)$ & $\lambda(\AA)$ & $W_{\mathrm{e}}(\mathrm{m} \AA)$ \\
\hline$\left[\mathrm{Fe}_{\mathrm{II}}\right]$ & 4905.35 & 4905.24 & 5 & 4905.32 & 238 \\
\hline$[\mathrm{Fe}$ II $]$ & 4923.92 & & & 4923.88 & 86 \\
\hline$[\mathrm{Fe} \mathrm{II}]$ & 4947.38 & & & 4947.38 & 64 \\
\hline$[\mathrm{Fe}$ II $]$ & 4950.74 & & & 4950.71 & 106 \\
\hline$[\mathrm{Fe}$ II $]$ & 4973.39 & & & 4973.37 & 124 \\
\hline$\left[\mathrm{Fe}_{\mathrm{II}}\right]$ & 4979.92 & 4979.93 & 9 & 4979.97 & 47 \\
\hline$[\mathrm{Fe} \mathrm{III}]$ & 4987.30 & & & - & $<5$ \\
\hline$[\mathrm{Fe} \mathrm{III}]$ & 5011.30 & & & 5011.32 & 32 \\
\hline$\left[\mathrm{Fe}_{\mathrm{I}}\right]$ & 5018.35: & & & 5018.40 & 141 \\
\hline$[\mathrm{Fe}$ II $]$ & 5020.23 & & & 5020.21 & 101 \\
\hline Si II & 5041.06 & 5040.98 & 57 & 5040.99 & 85 \\
\hline$\left[\mathrm{Fe}_{\mathrm{II}}\right]$ & 5043.52 & & & 5043.49 & 64 \\
\hline Si II & 5056.02 & 5055.98 & 182 & 5055.94 & 243 \\
\hline$[\mathrm{Mn}$ II] & 5086.84: & 5086.81 & 10 & & \\
\hline [Cr III] & 5107.89 & & & 5107.90 & 57 \\
\hline$\left[\mathrm{Fe}_{\mathrm{II}}\right]$ & 5111.62 & & & 5111.61 & 144 \\
\hline
\end{tabular}

the latter, so that microturbulence was only permitted to affect the line profiles. For reasons of computational speed we also used the standard TLusTy Opacity Distribution Functions rather than the more computationally demanding Opacity Sampling approach available in more recent versions of the code.

The methods used to determine the stellar effective temperature, surface gravity, and microturbulent velocity $\left(T_{\mathrm{eff}}, \log g\right.$, and $v_{\mathrm{t}}$ respectively) were similar to those adopted previously (see, for example, McErlean et al. 1999). Grids of model atmospheres were created for a range of values of $T_{\text {eff }}, \log g$, and $v_{\mathrm{t}}$, and metal abundances, and theoretical metal line equivalent widths obtained for all features reliably observed in the programme star, in addition to detailed hydrogen and helium line profiles. An iterative technique was then employed, where values of $\log g$ and $v_{\mathrm{t}}$ were assumed, and the temperature estimated using the constraint that the abundances deduced from different ionization stages of silicon ( $\mathrm{Si}$ III and $\mathrm{Si}$ IV) must be the same. For this combination of $T_{\text {eff }}$ and $v_{\mathrm{t}}, \log g$ was established by comparing the observed $\mathrm{H}_{\gamma}, \mathrm{H}_{\delta}$, and $\mathrm{H}_{\epsilon}$ profiles to the theoretical profiles. Although there is strong nebular emission in the cores of the Balmer lines of our PAGB candidates, the quality of the observational data allow the gravity to be estimated by fitting at least one of the stellar absorption line wings. The new gravity estimate was then used as the starting point in the next iteration to find $T_{\text {eff }}$, and the process repeated to convergence.

The microturbulent velocity was estimated using the requirement that the abundances derived from the observed $\mathrm{O}_{\text {II }}$ lines be independent of the line strength (i.e. a plot of abundance against line strength has a zero gradient). This led to further iterations of the entire procedure until convergence was obtained for $T_{\text {eff }}, \log g$, and $v_{\mathrm{t}}$. The spectrum of the standard star, HD 13841, was analysed using similar techniques and the adopted atmospheric parameters for all three stars are summarized in Table 3 . We note that the value of $v_{\mathrm{t}}$ for all stars is quite large, but the O II diagnostic showed this was the smallest acceptable value. In their non-LTE analysis, McErlean et al. (1999) also found supergiants with comparable atmospheric parameters (but at a different evolutionary stage to our PAGB candidates) that required similarly large values of $v_{\mathrm{t}}$ to be adopted.

Abundances for the other metals observed in the spectrum were determined by simple linear interpolation between points in our grids of model atmospheres. Previous experience has shows that the grid spacing used $(0.4$ dex increments in abundance) is sufficiently fine to ensure that no significant errors were introduced by the interpolation process. Abundances were calculated on a line-by-line basis, and then differential results obtained by simply comparing the abundance derived for the programme star to that found for the standard. The final absolute and differential (with respect to HD 13841) abundances for LSIV -12 111 and HD 314617 are shown in Tables 3 and 4 respectively.

\subsection{Analysis of the emission line spectra}

The spectroscopy for both LSIV -12 111 and HD 341617 was optimized in order to analyse the absorption line spectrum and hence utilized a narrow slit. Hence it is not possible to obtain reliable absolute fluxes for the emission lines. However using the equivalent widths listed in Table 2 and stellar continuum flux distributions for the atmospheric parameters deduced above, it is possible to deduce reliable emission line flux ratios. We note that this procedure corrects for both wavelength variations in the instrumental response and for interstellar absorption, while the relatively small wavelength range covered by our spectra resulted in the corrections being relatively minor.

The emission line spectrum of LSIV -12 111 has been previously discussed by CDMK93, but due to the quality of their observations only relatively strong lines were detected. From the $\left[\mathrm{S}_{\mathrm{II}}\right]$ doublet near $6720 \AA$ and the [O II] doublet near $3737 \AA$, logarithmic electron density estimates, $\log N_{\mathrm{e}}$ $\left(N_{\mathrm{e}}\right.$ in $\left.\mathrm{cm}^{-3}\right)$ of 3.5 and 3.7 , respectively, were deduced. Additionally the [N $\left.\mathrm{N}_{\mathrm{II}}\right]$ lines at $5755 \AA$ and approximately $6550 \AA$ were used to estimate a temperature, $T_{\mathrm{e}}=7800 \mathrm{~K}$. The latter estimate should be treated with some caution as the identification and measurement of the strength of the weaker line at $5755 \AA$ was marginal.

The relatively rich [Fe III] spectrum in HD 341617 provides a number of electron density diagnostics (Keenan et al. 2001), as do the [O $\left.\mathrm{O}_{\mathrm{II}}\right]$ lines in both stars. Unfortunately the limited spectral coverage does not provide an independent electron temperature diagnostic for HD 341617, and hence we have simply adopted the value found by CDMK93 for LSIV -12111 , i.e. $T_{\mathrm{e}}=7800 \mathrm{~K}$. We note that Parthasarthy et al. (2000) have derived a nebular temperature of $T_{\mathrm{e}}=10000 \mathrm{~K}$ for HD 341617 from $[\mathrm{Fe}$ II] line ratios, but this must be treated with caution given the uncertainties in atomic data for this ion (Ramsbottom et al. 2002). However the [O II] and [Fe III] line ratios employed here are relatively insensitive to temperature, and hence the density estimates discussed below are effectively independent of the adopted electron temperature.

For LSIV -12111, the line ratio for the [O II] doublet is 0.50 , which is in good agreement with that found by CDMK93. Adopting the calculations of Keenan et al. (1999) 
Table 3. Absolute metal abundances on a logarithmic scale with the abundance of hydrogen being 12.0 (i.e. $\left.\epsilon(X)=12+\log \frac{\mathrm{X}}{\mathrm{H}}\right)$ for LSIV -12111 and HD 341617 and the standard star, HD 13841. Errors are standard errors for $n$ measurements. Also listed are typical abundances for earlytype stars (see text for details) and for the sun (Anders \& Grevesse 1989).

\begin{tabular}{|c|c|c|c|c|c|c|c|c|}
\hline \multirow{2}{*}{$\begin{array}{l}\text { Parameter } \\
\text { element }\end{array}$} & \multicolumn{2}{|c|}{ LSIV -12 111} & \multicolumn{2}{|c|}{ HD 341617} & \multicolumn{2}{|c|}{ HD 13841} & \multirow{2}{*}{$\begin{array}{l}\text { B-type } \\
\epsilon(\mathrm{X})\end{array}$} & \multirow{2}{*}{$\begin{array}{l}\text { Sun } \\
\epsilon(\mathrm{X})\end{array}$} \\
\hline & $\epsilon(\mathrm{X})^{\dagger}$ & $n$ & $\epsilon(\mathrm{X})$ & $n$ & $\epsilon(\mathrm{X})$ & $n$ & & \\
\hline$T_{\text {eff }}$ & 20500 & & 20750 & & 19000 & & & \\
\hline $\log g$ & 2.35 & & 2.35 & & 2.65 & & & \\
\hline$v_{\mathrm{t}}$ & 20 & & 20 & & 20 & & & \\
\hline $\mathrm{C}$ & $7.50 \pm 0.24$ & 2 & 7.04 & 1 & $7.92 \pm 0.20$ & 2 & $7.8-8.2$ & 8.52 \\
\hline $\mathrm{N}$ & $7.65 \pm 0.04$ & 15 & $7.52 \pm 0.17$ & 7 & $8.07 \pm 0.07$ & 15 & $7.4-7.8$ & 8.01 \\
\hline $\mathrm{O}$ & $8.51 \pm 0.02$ & 49 & $8.35 \pm 0.04$ & 24 & $8.70 \pm 0.05$ & 49 & $8.4-8.7$ & 8.89 \\
\hline $\mathrm{Ne}$ & $8.17 \pm 0.04$ & 3 & & & $8.58 \pm 0.05$ & 3 & $8.0-8.1$ & 8.05 \\
\hline $\mathrm{Mg}$ & 6.76 & 1 & 6.32 & 1 & 6.80 & 1 & $7.1-7.4$ & 7.54 \\
\hline $\mathrm{Si}$ & $7.32 \pm 0.02$ & 4 & $7.07 \pm 0.06$ & 4 & $7.75 \pm 0.01$ & 4 & $7.2-7.5$ & 7.51 \\
\hline S & $7.40 \pm 0.14$ & 3 & $7.25 \pm 0.15$ & 3 & $7.73 \pm 0.10$ & 4 & $7.0-7.2$ & 7.17 \\
\hline
\end{tabular}

$\epsilon(\mathrm{X})=12+\log \frac{\mathrm{X}}{\mathrm{H}}$

Table 4. Differential metal abundances for LSIV-12111 and HD 341617 with respect to HD 13841. Errors are standard errors for $n$ measurements.

\begin{tabular}{llccc}
\hline \hline Element & \multicolumn{2}{c}{ LSIV -12111} & HD 341617 \\
& $\Delta\left[\frac{\mathrm{X}}{\mathrm{H}}\right]^{\dagger}$ & $n$ & $\Delta\left[\frac{\mathrm{X}}{\mathrm{H}}\right]$ & $n$ \\
\hline $\mathrm{C}$ & $-0.42 \pm 0.12$ & 2 & -0.47 & 1 \\
$\mathrm{~N}$ & $-0.43 \pm 0.07$ & 15 & $-0.57 \pm 0.27$ & 6 \\
$\mathrm{O}$ & $-0.18 \pm 0.05$ & 49 & $-0.43 \pm 0.10$ & 16 \\
$\mathrm{Ne}$ & $-0.41 \pm 0.10$ & 3 & - & - \\
$\mathrm{Mg}$ & -0.04 & 1 & -0.48 & 1 \\
$\mathrm{Si}$ & $-0.43 \pm 0.04$ & 4 & $-0.68 \pm 0.08$ & 4 \\
$\mathrm{~S}$ & $-0.33 \pm 0.11$ & 3 & $-0.48 \pm 0.23$ & 3 \\
\hline
\end{tabular}

$\dagger \Delta\left[\frac{\mathrm{X}}{\mathrm{H}}\right]=\epsilon(\mathrm{X})_{*}-\epsilon(\mathrm{X})_{\mathrm{HD} 13841}$.

leads to an electron density estimate of $\log N_{\mathrm{e}}=3.5$, in excellent agreement with that of CDMK93. For HD 341617, we list in Table 5 the observed line ratios and the implied electron densities using the calculations of Keenan et al. (1999, 2001) for [O II] and [Fe III], respectively.

An inspection of Table 5 reveals that most of the [Fe III] line ratios indicate electron densities that are consistent, and which are also in agreement with the value determined from [O II]. By contrast, the [Fe III] ratios 4881/4658, 4987/4658 and $5011 / 4658$ all imply unrealistically high densities. However we note that the lines at 4881,4987 and $5011 \AA$ arise from ${ }^{5} \mathrm{D}-{ }^{3} \mathrm{H}$ and ${ }^{5} \mathrm{D}-{ }^{3} \mathrm{P}$ transitions within the $3 \mathrm{~d}^{6}$ configuration, while all other [Fe III] lines (which provide consistent $N_{\mathrm{e}}$ estimates) are due to ${ }^{5} \mathrm{D}-{ }^{3} \mathrm{~F}$ transitions within the $3 \mathrm{~d}^{6}$ configuration. Hence we postulate that the discrepancies are due to errors in the atomic data (in particular electron excitation rates) for the ${ }^{5} \mathrm{D}-$ ${ }^{3} \mathrm{H}$ and ${ }^{5} \mathrm{D}-{ }^{3} \mathrm{P}$ transitions. Interestingly, Esteban et al. (1998) discuss similar problems for [Fe III] in the Orion Nebula, with ratios involving ${ }^{5} \mathrm{D}-{ }^{3} \mathrm{~F}$ lines showing very good agreement between theory and observation, but large discrepancies being present when other transitions are considered. We note that new electron impact excitation rate calculations are currently underway for Fe III (see McLaughlin et al. 2002 for more details),
Table 5. Emission line ratios and electron density estimates for HD 341617.

\begin{tabular}{|c|c|c|c|}
\hline Ion & $\overline{\text { Ratio }}$ & Observed & $\begin{array}{c}\log N_{\mathrm{e}} \\
\left(N_{\mathrm{e}} \text { in } \mathrm{cm} \mathrm{s}^{-3}\right)\end{array}$ \\
\hline$\left[\mathrm{O}_{\mathrm{II}}\right]$ & $3729 / 3726$ & 0.34 & 4.3 \\
\hline$[\mathrm{Fe}$ III] & $4734 / 4658$ & 0.21 & 4.5 \\
\hline [Fe III] & $4702 / 4658$ & 0.37 & 4.1 \\
\hline$[\mathrm{Fe}$ III] & $4769 / 4658$ & 0.15 & 4.5 \\
\hline [Fe III] & $4778 / 4658$ & 0.07 & 4.0 \\
\hline$[\mathrm{Fe}$ III] & $4881 / 4658$ & 0.24 & $>5.5$ \\
\hline [Fe III] & $4987 / 4658$ & $<0.04$ & $>5.5$ \\
\hline [Fe III] & $5011 / 4658$ & 0.27 & $>5.5$ \\
\hline
\end{tabular}

and we intend to undertake a re-analysis of the HD $341617 \mathrm{ob}-$ servations (plus other spectra) once these new atomic data are available.

\section{Discussion}

Below we first discuss the stellar properties of our two stars and then compare their chemical compositions with those found for other PAGB candidates. Finally we use the stellar and circumstellar properties to discuss the evolutionary status of our targets.

\section{1. $L S I V-12111$ and $H D 341617$}

The stellar spectra of both LSIV-12111 and HD 341617 have been previously analysed using LTE model atmosphere techniques. For LSIV -12 111, the analyses of MCDK92 and CDMK93 were based on relatively low quality spectroscopic data, whilst the analysis by Mooney et al. (2002) of several field PAGB candidates used effectively the same dataset for HD 341617 as discussed here. Additionally Parthasarthy et al. (2000) have analysed moderate resolution $(0.3 \AA)$ spectroscopy for HD 341617 obtained at the Isaac Newton Telescope. For both targets, all the LTE analyses yielded effective temperature estimates approximately 2000 to $3000 \mathrm{~K}$ higher than those found here. This is unlikely to be due to the effects of line 
blanketing, as the LTE analyses used fully line blanketed models, which normally lead to smaller effective temperature estimates (see, for example, McErlean et al. 1999). Hence the differences are probably due to non-LTE effects. Additionally the gravities deduced here are significantly lower than those from the LTE analyses. This is particularly the case for HD 314617 , where Parthasarthy et al. deduced a logarithmic gravity of 3.0 dex, which is larger by 0.65 dex than that found here. Their value was based on the diffuse helium lines as the spectral resolution of their observations was insufficient to separate the absorption profile from the nebula emission. For the other LTE analyses that utilize the hydrogen lines the differences in the gravity estimates are approximately 0.4 dex. These differences will partially reflect the values adopted for the effective temperatures but will also be due to the well known increase in the strength of Balmer lines when non-LTE effects are included (see, for example, Mihalas \& Auer 1970). Interestingly the LTE and non-LTE analyses yield similar estimates for the microturbulence in both stars. This contrasts with some other studies where lower microturbulences have been found in a non-LTE regime (see, for example, Gies \& Lambert 1992 and Vrancken et al. 2000).

It is also possible to compare the chemical compositions deduced from the different analyses. The individual abundances estimates from the LTE and non-LTE analyses typically differ by 0.2 to 0.4 dex but are as large as 0.8 dex - note that Parthasarthy et al. (2000) do not quote absolute abundances. In general the non-LTE approach yields lower abundance estimates and this is consistent with the observations that nonLTE effects generally increase line strengths (see, for example, Becker \& Butler 1988, 1989, 1990). In the case of C and S, the differences are in the opposite sense. For the former, this is again consistent with several studies (Lennon 1983; Eber \& Butler 1988; Sigut 1996) that have shown that non-LTE effects weaken $\mathrm{C}_{\text {II }}$ line strengths, due to overionization and $\mathrm{C}_{\text {II }}$ being a minority ionization state. The differences for $\mathrm{S}$ (based mainly on S III lines) are significant (0.5-0.8 dex) and it is unclear whether this is predominantly due to non-LTE effects or some other mechanism (for example, differences in the adopted oscillator strengths or use of different sets of lines). In general it would be expected that the absolute abundances presented here will be more reliable than the previous LTE calculations, although for these relative low gravity stars there remains significant uncertainties, similar to those discussed by McErlean et al. (1999).

For HD 341617, we can also compare the differential abundances obtained from LTE and non-LTE analyses (CDMK93 did not undertake a differential analysis for LSIV -12 111). For the analysis of Mooney et al. (2002) the same standard was used and hence the comparison should be particularly worthwhile. In this case the differential abundances from the two approaches agree better than the absolute abundances, with differences being typically 0.2 to $0.3 \mathrm{dex}$. This is not surprising as first order systematic errors in the analysis of the programme star and the standard should cancel. The differences between the two analyses show no clear pattern and probably reflect the different estimates of the atmospheric parameters and variations in the shapes of the LTE and non-LTE curves of growth.
Parthasarthy et al. (2000), as noted previously, only quote relative abundances in their paper, but do not seem to identify what these have been measured relative to. Since they used SYNSPEC, we make the assumption that they are quoting abundances relative to the defaults used in that program, which are solar (Grevesse \& Sauval 1998). We note that it is therefore possibly not appropriate to use their abundances in the context of B-type stars. For example, Parthasarthy et al. deduce a $\mathrm{C}$ underabundance larger by more than 1.0 dex than our non-LTE result. However as can be seen from Table 3, non-LTE calculations for B-type stars yield lower carbon abundances than are found in the sun, with LTE calculations leading to even smaller estimates. Hence the comparison of the differential abundance estimates of Parthasarthy et al. and the values deduced here may be complicated by the different choice of standard. However apart from $\mathrm{C}$ and $\mathrm{S}$ the different estimates are in reasonable agreement with discrepancies being less than 0.2 dex. Again we would expect that the values presented here, which are based on non-LTE methods with a standard that has suitable atmospheric parameters would be the more reliable.

\subsection{Comparison with other stellar chemical compositions}

In Table 3, we list, as well as absolute abundance estimates for our targets, typical values for B-type stars and the Sun (Anders $\&$ Grevesse 1989). As discussed by Vrancken et al. (2000), there have been several non-LTE analyses of bright relatively unevolved B-type stars (for example, Gies \& Lambert 1992; Kilian 1992; Vrancken et al. 1997), which have yielded a range of chemical compositions. Hence rather than give a single value for each element, in Table 3, we list the range of abundances implied by these analyses. The absolute abundances deduced for our bright supergiant, HD 13841, are generally consistent with these ranges although there are significant differences for some of the heavier elements. It is unlikely that these are due to mixing of nucleosynthetic material to the surface, as we would then expect to see significant changes in the CNO abundances. Rather, they probably reflect the relatively small number of lines considered together with the limitations of using classical non-LTE techniques for stars with low surface gravities. In particular, we note that the $\mathrm{N}$ abundance in HD 13841 appears to be normal and this is consistent with the conclusion of McErlean et al. (1999) that the surface composition of this star had not been significantly affected by nucleosynthetic processing. Hence we believe that HD 13841 may provide a reliable baseline composition for the differential abundance analysis.

The differential chemical composition for both PAGB candidates are shown in Table 4. In general they are consistent with uniform metal underabundances of approximately $-0.35 \mathrm{dex}$ in LSIV -12111 and -0.50 dex in HD 341617. Indeed the only estimate to differ by more than 0.2 dex from these values is that for Mg in LSIV - 12111 which is based on one line. If we assume that these objects are old and that their original chemical composition was metal deficient, then it would appear that their atmospheres have not been significantly affected by dredgeup processes that might have been expected to occur on the 
red-giant and asymptotic-giant-branches. However, it should be noted that if the atmosphere of the standard HD 13841 had been contaminated by nucleosynthetic material processed by the CNO-bicycle, this would mask a similar level of contamination in our PAGB candidates - for reasons outlined above, we believe that this is unlikely but not impossible.

One disappointment of the current investigation was that it was not possible to estimate reliable $\mathrm{Fe}$ abundances in our PAGB candidates. Their effective temperatures implies that both their Fe II and Fe III spectra will be relatively weak with the latter being the stronger. Unfortunately the strongest Fe III absorption line at approximately $4419 \AA$ is badly blended with an emission feature (see Table 2), whilst other features could not be identified. Upper limits on the equivalent widths imply that $\mathrm{Fe}$ is underabundant in our PAGB candidates but the constraint on the abundances is not particularly stringent and is consistent with the mean metallicities of the targets.

Recent abundance analyses of PAGB stars reported in the literature reveal a rather complex picture (see van Winckel et al. 1998 for a recent review). Indeed, van Winckel (1997) differentiates between 3 classes of objects based upon observations of optically bright, F-type PAGBs. These can be summarized as follows:

- C-rich, overabundant in s-process elements and, in the example of HD 187885 (van Winckel et al. 1996), a suprasolar He abundance. Such an abundance pattern is clear evidence for an efficent third dredge-up episode;

- A second class of PAGB stars include objects like HD 52961 (Waelkens et al. 1991; van Winckel et al. 1995) where $\mathrm{Fe}$ is underabundant by approximately 5 orders of magnitude and $\mathrm{CNO} \& \mathrm{~S}$ abundances are approximately Solar;

- The remaining objects display a general trend that is indicative of an old population, viz. atmospheres that contain a mixture of CNO-cycled material and He-burning products (Luck 1993; van Winckel 1997). Additionally, some stars display higher $\mathrm{N}$ abundances; however, the $s$-process elements do not appear to be enhanced.

Historically, high-latitude, B-type PAGB candidates were found to display a significantly different abundance pattern to that of the optically bright, F-type PAGBs, viz. model atmosphere analyses of the former group consistently revealed a striking C deficiency (see Conlon et al. 1994). In many other aspects, these B-type objects are not dissimilar to the third group of PAGB objects discussed by van Winckel (1997). For example, some of the field PAGB stars (e.g. LB 3219) considered by MCDK92 and the globular cluster star Barnard 29 (Conlon et al. 1994; Moehler et al. 1998), exhibit significant $\mathrm{N}$ enhancements (relative to other metals) and these have been interpreted as evidence for the dredge-up of CNO-processed material. Analyses of other PAGB-candidates (Moehler \& Heber 1998; Napiwotzki et al. 1993) show relatively normal N abundances (compared with other metals). However, if the third dredge-up indeed occurred in these objects, then one has to invoke scenarios where the $\mathrm{C}$ abundance remained low (see MCDK92). This has led van Winckel (1997) to conclude that the abundance patterns do not support a common origin for the optically bright, F-type and halo B-type PAGBs. It is worthwhile noting one exception in the sample of optically bright, F-type PAGB stars studied by van Winckel; HD 107369 shows evidence of a $\mathrm{C}$ deficiency. Indeed, we have presented the abundance pattern for this object in Table 6 together with the key element abundances (viz, C, N, O, S, \& Fe) found for the B-type, PAGB star ZNG-1 in the Globular cluster M 10 (Mooney et al. 2002) - the agreement is remarkable Furthermore, HD 107369 possesses the largest surface gravity (corresponding to a lower mass) of stars in the van Winckel sample of PAGBs. This is interesting as the majority of halo, B-type PAGBs which exhibit large $\mathrm{C}$ deficiencies lie along the lower mass PAGB tracks (see Schönberner 1983, 1987).

Given these discussions, we have attempted to compare the results obtained for LSIV -12 111 and HD 341617 with the studies of the cool PAGBs. However, PAGB/Population II abundances are generally compared with Solar values and are given relative to $\mathrm{Fe}$. Unfortunately, it was not possible to derive Fe abundance estimates for LSIV - 12111 and HD 341617. Additionally, significant differences exist between the absolute abundances derived for the Sun and normal, Population I Btype stars in the Solar neighnourhood (see Gies \& Lambert 1992). Thus, we believe our differential abundances presented in Table 4 to be more reliable. It was not possible to obtain corresponding differential abundances of the optically bright, Ftype PAGBs relative to normal, Population I F-type supergiants of similar atmospheric parameters (as van Winckel 1997 did not present abundance information for the latter). Therefore, in Table 6 the differential abundance estimates presented for the cool PAGBs are simply those of van Winckel (1997) measured relative to $\mathrm{H}$ and compared with the Sun. Furthermore, we have attempted to undertake a comparison with Population II abundance values by transforming the results of Caretta et al. (2000) into abundance ratios measured relative to hydrogen. In this analysis, we have adopted a $\log L / L_{\odot}$ value of 4.0 and a mean metallicity of -0.5 dex in order to extract typical Population II abundance ratios for objects of comparable luminosity and metallicity. The results are presented in Table 6 .

The comparisons in Table 6 should be treated with caution as we are not necessarily comparing "like with like". However, it is very interesting that the abundance patterns observed for LSIV -12 111 and HD 341617 are very similar to that observed for the optically bright, F-type PAGB stars SAO 239853 and SAO 173329 respectively. Hence, these high mass (see Sect. 4.3), B-type PAGBs with circumstellar material may be the hotter analogues of the third group of F-type PAGBs discussed by van Winckel et al. (1998), indicating a common origin for such objects. However, the definitive test would be the determination of Fe abundances for LSIV -12 111 and HD 341617.

\subsection{Evolutionary status}

The mass of our targets can be estimated assuming that they are at a PAGB evolutionary stage and using the evolutionary tracks of Schönberner $(1983,1987)$. Both stars lie close to the 0.846 solar mass track (see, for example, Fig. 2 of 
Table 6. Comparison of LSIV - 12111 and HD 341617 with other PAGB objects.

\begin{tabular}{llllllllll}
\hline \hline \multirow{2}{*}{ Element } & $\mathrm{LSIV}^{1}$ & $\mathrm{HD}^{1}$ & $\mathrm{HD}^{2}$ & $\mathrm{SAO}^{2}$ & $\mathrm{SAO}^{2}$ & $\mathrm{HD}^{2}$ & Pop II $^{3}$ & $\mathrm{M} \mathrm{10}^{4}$ & $\mathrm{HD}^{2}$ \\
& -12111 & 341617 & 133656 & 173329 & 239853 & 131356 & & ZNG-1 & 107369 \\
\hline $\mathrm{C}$ & -0.4 & -0.5 & -0.5 & -0.5 & -0.4 & -0.4 & & -2.5 & $<-1.3$ \\
$\mathrm{~N}$ & -0.4 & -0.6 & -0.2 & -0.5 & -0.3 & -0.2 & +0.5 & -0.7 & -0.7 \\
$\mathrm{O}$ & -0.2 & -0.4 & -0.1 & & -0.2 & -0.6 & -0.2 & -0.9 & -1.1 \\
$\mathrm{~S}$ & -0.3 & -0.5 & -0.3 & -0.7 & -0.4 & -0.6 & -0.2 & -0.9 & -1.0 \\
$\alpha$ & -0.3 & -0.6 & -0.5 & -0.7 & -0.4 & -0.6 & -0.4 & -1.0 & -0.9 \\
$\mathrm{Fe}$ & & & -0.7 & -0.8 & -0.8 & -0.7 & -0.4 & -0.8 & -1.1 \\
\hline
\end{tabular}

${ }^{1}$ This work.

${ }^{2}$ Optically bright, F-type PAGB stars (Van Winckel 1997).

${ }^{3}$ Population II giants (Carretta et al. 2000).

${ }^{4}$ Globular cluster, B-type PAGB (Mooney et al. 2003).

Mooney et al. 2002), implying that they are relative high mass and will be evolving rapidly. We note that these mass estimates are significantly larger than those found by CDMK93 for LSIV -12 111 or implied by the atmospheric parameters of Parthasarthy et al. (2000) for HD 341617. This is due principally to the lower gravities deduced using the non-LTE calculations adopted here. However our mass estimate for HD 341617 is similar to that found by Mooney et al. (2002), as although they deduced a higher surface gravity, they also estimated a higher effective temperature. Both stars have emission arising from circumstellar material, whilst LSIV -12 111 has an infrared excess due to dust emission (CDMK93). These properties are all compatible with a high mass, rapidly evolving PAGB evolutionary status.

As discussed by Parthasarthy et al. (2000) and Mooney et al. (2002), our targets lie in the same part of the gravityeffective temperature diagram as other PAGB stars, viz. PHL 1580, LB 3219 (MCDK92), CPD -61 455 (Hambly et al. 1996), CD -41 13967 (McCarthy et al. 1991), HD 172324 (Arellano Ferro et al. 2001), PG 1323-086 (Moehler \& Heber 1998) and BD +33 2642 (Napiwotzki et al. 1993). All these latter stars have smaller inferred masses, which is consistent with their larger timescales for evolution from the AGB stars into Planetary Nebula. Apart from its higher mass, LSIV -12 111 can also be distinguished from these other stars in that it would appear to be part of a binary system from the radial velocity variation in its absorption line spectra. CDMK93 and Conlon et al. (1993b) have discussed the near and far-infrared flux excess observed in LSIV -12111 and found it compatible with dust emission at a temperature of approximately $150 \mathrm{~K}$. In particular, there is no evidence for a significant contribution from a cool stellar companion. However the central stars of Planetary Nebulae are often members of binary systems (see, for example Bond 2000). Additionally Pollacco \& Sorenson (private communication) have found radial velocity variations in a significant fraction of their sample of Planetary Nebulae, with indications that some of these central stars are also single-lined spectroscopic binaries.

As discussed above, the relatively large masses estimated for our targets imply that they should evolve rapidly from the AGB, the discussion of Blöcker \& Schönberner (1990) indicating a period of approximately 100-200 years. There is some evidence for circumstellar material from the emission line spectra and from the observation that both objects are associated with IRAS sources. However it is surprising that there is not more evidence of the substantial circumstellar envelope that is found to obscure many PAGB stars (see, for example, Sahia et al. 1998, 1999; Ueta et al. 2000; Trammell \& Goodrich 2002). This problem could be ameliorated if the masses for our objects had been overestimated. We believe that the quality of our observational data allows us to distinquish between models with values of the logarithmic gravity differing by 0.2 dex. Additionally there may be errors due to the assumptions made in the model atmosphere analysis. However given the sophistication of the non-LTE methods adopted here and their success in modelling the spectra of other stars (see, for example, Hubeny et al. 1998), we would not expect these to be large. However, for example, adopting an uncertainty of upto $0.3 \mathrm{dex}$ in the gravity estimates would allow us to place both objects near the 0.565 solar mass track of Schönberner (1983), which would increase the time since leaving the AGB to approximately 3000 years.

A second explanation is that our targets have been misidentified. In such circumstance, the other possibilities would appear to be that they are normal young supergiants or have evolved directly off the extended horizontal branch (EHB). If the former, their distances would become very large distances (of the order of 20-30 kpc) and given their high galactic latitudes $\left(29^{\circ}\right.$ for LSIV -12111 and $\left.20^{\circ} \mathrm{HD} 341617\right)$ they would be situated more than $10 \mathrm{kpc}$ from the galactic plane. Additionally as discussed by both CDMK93 and Parthasarathy et al. (2000) their IRAS colours are similar to those found for planetary nebulae.

Dorman et al. (1993) have discussed the evolution of stars from the EHB. In particular, if they retain sufficient envelope mass they can start to ascend the AGB but will evolve off it prior to the onset of thermal pulse. Dorman et al. characterize such objects as post-early AGB stars and find they are generally less luminous $\left(\log L / L_{\odot} \leq 3\right)$ than PAGB stars. From our atmospheric parameters and inferred masses, the luminosities of our objects are approximately $10^{4} L_{\odot}$, which is inconsistent with this scenario. Hence we conclude that our targets are probably PAGB stars, although how the significant circumstellar material that should have been ejected on the AGB, has dissipated, remains unclear. 
The emission line spectra for our targets have been discussed in Sect. 3.2. Although the spectra are similar in the two stars, there are differences in, for example, the strengths of the forbidden lines. These will be due to a combination of the different plasma parameters and the size of the emitting volume (note that as emission line fluxes are measured relative to a normalized stellar continuum, the distance of the stars should not directly affect these values). The plasma parameters that we have deduced are compatible with those previously estimated by CDMK93 and Parthasarthy et al. (2000), and as discussed by these authors consistent with the expected environment around a PAGB star. CDMK93 used the spatial extent of the emission lines (normal to the dispersion direction and relative to that of the stellar continuum) to estimate the angular size of the emission around LSIV -12111 as $1.2 \pm$ 0.2 arcsec. Inspection of the two dimensional echelle spectra of HD 341617 indicate that the strong emission may have a greater spatial extent than the stellar continuum. However during read-out of the CCD, two pixels had been binned together along this axis, effectively reducing the spatial resolution. Attempts to measure the spatial extent of these emission lines was not convincing, although an upper limit of 0.25 arcsec could be established. This is significantly smaller than that found for LSIV -12111 but it should be noted that the density of the circumstellar material around HD 341617 is an order of magnitude larger than that around LSIV -12111; as the ionizing radiation emitted by the two stars will be similar (given their atmospheric parameters), this will reduce the spatial extent of the emission.

Our nebula analyses have been limited by the relatively restricted wavelength coverage of our spectra. For example, no temperature diagnostic was available for HD 341617 and a value of $T_{\mathrm{e}}$ had to be assumed (Sect. 3.2). Additional observations with a more extensive wavelength coverage are planned to allow detailed nebular parameters to be determined. In particular, nebular abundances may be compared with photospheric values to estimate the amount and chemical composition of material lost by the star during nebular formation.

Acknowledgements. CJM would like to thank the Department of Education and Learning (DEL) for Northern Ireland and the Isaac Newton Group, La Palma for the award of a CAST research studentship. WRJR and RSIR acknowledge financial support from the UK Particle Physics and Astronomy Research Council (PPARC). We are grateful to Peter van Hoof for the use of his Atomic Line List website.

\section{References}

Aller, L. H., \& Hyung, S. 1995, MNRAS, 276, 1101

Alves, D. R., Bond, H. E., \& Onken, C. 2001, AJ, 121, 318

Anders, E., \& Grevesse, N. 1989, Geochim. Cosmochim. Acta, 53, 197

Arellano Ferro, A., Giridhar, S., \& Mathias, P. 2001, A\&A, 368, 250

Asplund, M., Lambert, D. L., Kipper, T., et al. 1999, A\&A, 343, 507

Becker, S. R., \& Butler, K. 1988, A\&A, 201, 232

Becker, S. R., \& Butler, K. 1989, A\&A, 209, 244

Becker, S. R., \& Butler, K. 1990, A\&A, 235, 326
Blöcker, T., \& Schönberner, D. 1990, A\&A, 240, L11

Bond, H. E. 2000, ed. J. H. Kastner, N. Soker, \& S. Rappaport, APS Conf. Ser., 199, 115

Butler, K. 1984, Thesis, University of London

Carretta, E., Gratton, R. G., \& Sneden, C. 2000, A\&A, 356, 238

Conlon, E. S., Dufton, P. L., McCausland, R. J. H., et al. 1993, ApJ, 408, 593

Conlon, E. S., Dufton, P. L., Keenan, F. P., et al. 1993, A\&A, 272, 243

Conlon, E. S., Dufton, P. L., \& Keenan, F. P. 1994, A\&A, 290, 897

Dorman, B., Rood, R. T., \& O’Connell, R. W. 1993, ApJ, 419, 596

Dyer, K. Y., Goss, W. M., \& Kembell, A. J. 2001, AJ, 121, 2743

Eber, F., \& Butler, K. 1988, A\&A, 202, 153

Evans, A., Geballe, T. R., Tyne, V. H., et al. 2002, MNRAS, 332, L69

Giddings, J. R. 1981, Thesis, University of London

Gies, D. R., \& Lambert, D. L. 1992, ApJ, 387, 673

Gledhill, T. M., Bians, I., \& Yates, J. A. 2002, MNRAS, 332, L55

Grevesse, N., \& Sauval, A. J. 1998, Space Sci. Rev., 85, 161

Hambly, N. C., Keenan, F. P., Dufton, P. L., et al. 1996, MNRAS, 278, 811

Hambly, N. C., Rolleston, W. R. J., Keenan, F. P., \& Saffer, R. A. 1997, ApJS, 111, 419

Howarth, I. D., Murray, J., Mills, D., et al. 1996, staRlink User Note SUN 50, Rutherford Appleton Laboratory/CCLRC

Hrivnak, B. J. 1997, in Planetary Nebulae, ed. H. J. Habing, \& H. J. G. L. M. Lamers (Dordrecht: Kluwer), IAU Symp., 190, 303

Hubeny, I. 1988, Comp. Phys. Comm., 52, 103

Hubeny, I., \& Lanz, T. 1995, ApJ, 439, 875

Hubeny, I., Heap, S. R., \& Lanz, T. 1998, in Boulder-Munich: Properties of Hot, Luminous Stars, ed. I. D. Howarth, ASP Conf. Ser., 131, 108

Hyung, S., Aller, L. H., \& Feibelman, W. A. 1994, MNRAS, 269, 975

Hyung, S., Keyes, D. D., \& Aller, L. H. 1995, MNRAS, 272, 49

Hyung, S., Aller, L. H., Feibelman, et al. 1994, MNRAS, 318, 77

Keenan, F. P., Aller, L. H., Bell, K. L., et al. 1999, MNRAS, 304, 27

Keenan, F. P., Aller, L. H., Ryans, R. S. I., et al. 2001, Proc. Natl. Acad. Sci. USA, 98, 9476

Kilian, J. 1992, A\&A, 262, 171

Kilkenny, D., \& Pauls, L. 1990, MNRAS, 244, 133

Kwok, S. 1993, ARA\&A, 31, 63

Kwok, S., Su, K. Y. L., \& Hrivnak, B. J. 1998, ApJ, 501, L117

Landsman, W., Moehler, S., Napiwotzki, R., et al. 1998, BAAS, 30, 896

Lennon, D. J. 1983, MNRAS, 205, 829

Lennon, D. J., Dufton, P. L., Mazzali, et al. 1996, A\&A, 314, 243

Luck, R. E., \& Bond, H. E. 1991, ApJS, 77, 515

Luck, R. E. 1993, in A Workshop on Luminous High-latitude stars, ed. D. D. Sasselov, ASP, 87

Magee, H. R. M., Dufton, P. L., Keenan, F. P., et al. 2001, MNRAS, 324,747

Massey, P. 1989, A Users Guide to CCD reduction with IRAF, NOAO

McCarthy, J. K., Rich, R. M., Decker, S. R., et al. 1991, ApJ, 371, 380

McCausland, R. J. H., Conlon, E. S., Dufton, P. L., et al. 1992, ApJ, 394, 298

McErlean, N. D., Lennon, D. J., \& Dufton, P. L. 1999, A\&A, 349, 553

McKenna, F. C., Keenan, F. P., Hambly, N. C., et al. 1997, ApJS, 109, 225

McLaughlin, B. M., Scott, M. P., Sunderland, A. G., et al. 2002, J. Phys. B, 35, 2755

Mihalas, D., \& Auer, L. H. 1970, ApJ, 160, 1161

Moehler, S., \& Heber, U. 1998, A\&A, 335, 985

Moehler, S., Landsman, W., \& Napiwotzki, R. 1998, A\&A, 335, 510

Mooney, C. J., Rolleston, W. R. J., Keenan, F. P., et al. 2001, MNRAS, 326,1101 
Mooney, C. J., Rolleston, W. R. J., Keenan, F. P., et al. 2002, MNRAS, 337,851

Mooney, C. J., Rolleston, W. R. J., Keenan, F. P., et al. 2003, A\&A, in press

Napiwotzki, R., Schönberner, D., \& Wenske, V. 1993, A\&A, 268, 653

Napiwotzki, R., Heber, U., Köppen, J. 1994, A\&A, 292, 239

Osterbrock, D. E., Tran, H. D., \& Veilleux, S. 1992, ApJ, 389, 305

Oudmaijer, R. D. 1996, A\&A, 306, 823

Oudmaijer, R. D., van der Veen, W. E. C. J., Waters, L. B. F. M., et al. 1992, A\&AS, 96, 625

Oudmaijer, R. D., Waters, L. B. F. M., van der Veen, W. E. C. J., et al. 1995, A\&A, 299, 69

Parthasarathy, M., Garcia-Lario, P., Sivarani, T., et al. 2000, A\&A, 357 241

Ramsbottom, C. A., Scott, M. P., Bell, K. L., et al. 2002, J. Phys. B, 35,3451

Rauch, T., Heber, U., \& Werner, K. 2002, A\&A, 381, 1007

Reddy, B. E., Lambert, D. L., Gongalez, G., et al. 2002, ApJ, 564, 482

Sahai, R., Trauger, J. T., Watson, A. M., et al. 1998, ApJ, 493, 301

Sahai, R., Zijlstra, A., Bujarrahal, V., et al. 1999, AJ, 107, 1408

Schönberner, D. 1983, ApJ, 272, 708
Schönberner, D. 1987, in Late Stages of Stellar Evolution, ed. S. Kwok, \& S. R. Pottasch (Reidel, Dordrecht), 359

Shames, P. M. B., \& Tody, D. 1986, A Users Introduction to the IRAF Command Language, Version 2.3, NOAO

Sigut, T. A. A. 1996, ApJ, 473, 452

Su, K. Y. L., Volk, K., Kwok, S., et al. 1998, ApJ, 508, 744

Trammell, S. R., \& Goodrich, R. W. 2002, ApJ, 579, 688

van Winckel, H. 1997, A\&A, 319, 561

van Winckel, H., Decin, L., \& Waelkens, C. 1998, IAUS, 189, 176

van Winckel, H., \& Reyniers, M. 2000, A\&A, 354, 135

van Winckel, H., Waelkens, C., \& Waters, L. B. F. M. 1995, A\&A, 293, L25

van Winckel, H., Waelkens, C., \& Waters, L. B. F. M. 1996, A\&A, 306, L37

Vrancken, M., Hensberge, H., David, M., et al. 1997, A\&A, 320, 878

Vrancken, M., Lennon, D. J., Dufton, P. L., et al. 2000, A\&A, 358, 639

Uesta, T., Meixner, M., \& Bobrowsky, M. 2000, ApJ, 528, 861

Waelkens, C., van Winckel, H., Bogaert, E., et al. 1991, A\&A, 251, 495 